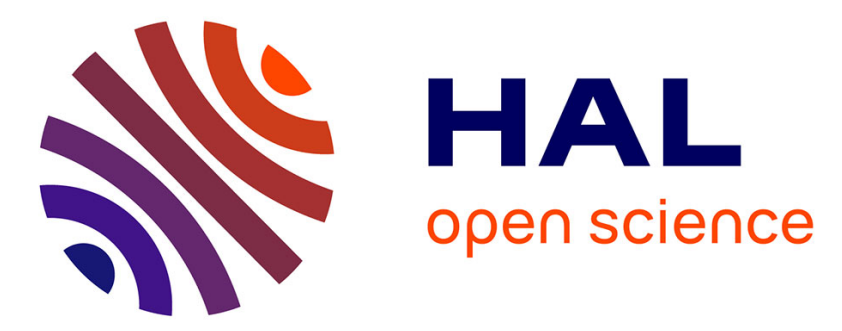

\title{
A comparative study of Rayleigh and Raman depolarized light scattering by a pure fluid of isotropic molecules
}

\author{
M. Thibeau, A. Gharbi, Y. Le Duff, V. Sergiescu
}

\section{- To cite this version:}

M. Thibeau, A. Gharbi, Y. Le Duff, V. Sergiescu. A comparative study of Rayleigh and Raman depolarized light scattering by a pure fluid of isotropic molecules. Journal de Physique, 1977, 38 (6), pp.641-651. 10.1051/jphys:01977003806064100 . jpa-00208623

\section{HAL Id: jpa-00208623 https://hal.science/jpa-00208623}

Submitted on 1 Jan 1977

HAL is a multi-disciplinary open access archive for the deposit and dissemination of scientific research documents, whether they are published or not. The documents may come from teaching and research institutions in France or abroad, or from public or private research centers.
L'archive ouverte pluridisciplinaire HAL, est destinée au dépôt et à la diffusion de documents scientifiques de niveau recherche, publiés ou non, émanant des établissements d'enseignement et de recherche français ou étrangers, des laboratoires publics ou privés. 


\title{
A COMPARATIVE STUDY OF RAYLEIGH AND RAMAN DEPOLARIZED LIGHT SCATTERING BY A PURE FLUID OF ISOTROPIC MOLECULES
}

\author{
M. THIBEAU
}

L.E.I.M.D.L. Faculté des Sciences d'Angers, 49045 Angers Cedex, France

\section{A. GHARBI, Y. LE DUFF}

Département de Recherches Physiques (*), Université de Paris VI, 75230 Paris Cedex, France

and

\section{SERGIESCU}

Faculté des Sciences d'Angers, 49045 Angers Cedex, France

et Département de Recherches Physiques (*), Université de Paris VI, 75230 Paris Cedex, France

(Reçu le 19 janvier 1977, accepté le 23 février 1977)

\begin{abstract}
Résumé. - On présente une théorie générale de l'effet de dépolarisation DID dans la diffusion Rayleigh et Raman, en réduisant le problème au calcul de certaines corrélations de position (binaires, ternaires et quaternaires). La dépendance taux de polarisation-densité est explicitée à basse et à haute densité. Le problème de l'interpolation aux densités intermédiaires est discuté en relation avec des mesures récentes.
\end{abstract}

\begin{abstract}
A general treatment of the depolarizing DID effect in Rayleigh and Raman light scattering is given in terms of binary, ternary, and quaternary position correlations. Explicit formulae are obtained at low and high densities for the density dependence of the depolarization ratio. The moderate densities case is discussed in relation with some recent observations.
\end{abstract}

1. Introduction. - Depolarized Rayleigh or Raman scattering by a pure fluid of isotropic molecules, as compared with polarized scattering, is a secondary effect resulting from the local electrical anisotropy due to the collisions of the molecules or, in more abstract terms, to the fluctuations of their positions. Molecular collisions involve an anisotropic modification of the individual polarizability of the molecules that may be real, when due to the electron density distortion, or apparent, when associated with the local field fluctuations (dipole induced dipole, or DID, effect).

In this paper we give a comparative treatment of the DID depolarized scattering in both the Rayleigh and Raman cases. We deal with the Raman vibration effect associated with a total symmetric normal mode, so that the depolarized scattering of the isotropic molecule is entirely of a collisional nature.

In contrast with the much investigated depolarized Rayleigh scattering [1-3], previous studies of the

$\left.{ }^{*}\right)$ Laboratoire associé au C.N.R.S. depolarized Raman scattering were generally limited to low density gases [4]. For both Raýleigh and Raman scattering we give in $\S 2$ a general treatment which reduces the problem to the calculation of certain binary, ternary, and quaternary position correlations. The computation is carried out in the low density limit and also, with the aid of the (partially occupied) lattice model, in the high density limit ( $\$ 3$ and $\S 4$ ). The problem of the interpolation at moderate densities is briefly dealt with in $\S 5$ and a preliminary discussion in relation with some recent experimental results is given in $\S 6$.

2. General theory. - In the following the incident monochromatic light wave is supposed to be polarized along $\mathrm{Oz}$ (vertical polarization) and to propagate along $O x$. The scattered light propagates along $O y$ and one can alternatively measure the vertical $O z$ polarized component (polarized or VV scattering) or/and the horizontal $O x$-polarized component (depolarized or VH scattering) (Fig. 1). The four scattered intensities being respectively $I_{\mathrm{VV}}^{\text {Ray }}, I_{\mathbf{V H}}^{\text {Ray }}, I_{\mathrm{VV}}^{\mathrm{Ram}}, I_{\mathbf{V H}}^{\mathrm{Ram}}$, 


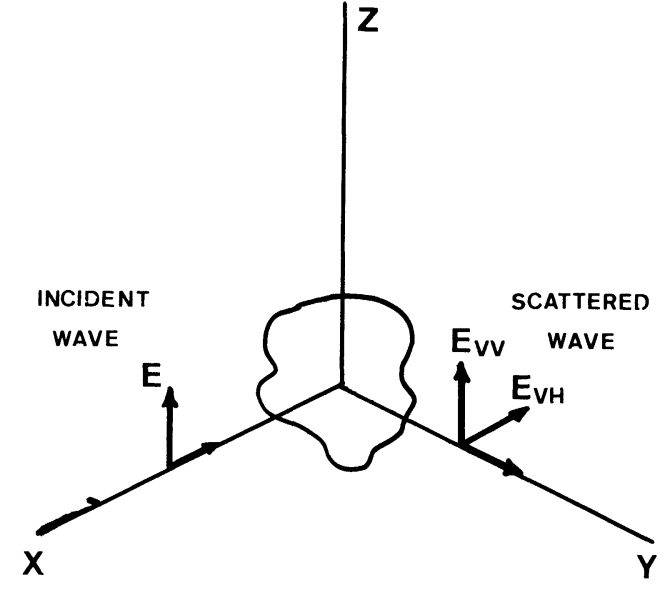

Fig. 1. - Polarization of the electric fields in the light scattering experiment.

the depolarizing effect of the intermolecular correlations is characterized by the depolarization ratios $\eta^{\text {Ray }}=I_{\mathrm{VH}}^{\text {Ray }} / I_{\mathrm{VV}}^{\text {Ray }}$ and $\eta^{\text {Ram }}=I_{\mathrm{VH}}^{\mathrm{Ram}} / I_{\mathrm{VV}}^{\mathrm{Ram}}$. Our system is a gas of $N$ identical isotropic molecules in a volume $V$.

In DID theory the polarizability $\alpha^{i}$ of an isotropic molecule $i$ is a scalar quantity not depending on the other molecules. In the classical approximation, or in the quantum adiabatic approximation, $\alpha^{i}$ is a function of the positions of the atoms forming the molecule $i$, i.e. of the normal coordinates $Q^{i}$, where
$Q^{i}$ is a synthetic notation for the ensemble of the normal coordinates belonging to this molecule :

$$
\alpha^{i}=\alpha\left(Q^{i}\right)=\alpha_{0}^{i}+\left(\frac{\partial \alpha^{i}}{\partial Q^{i}}\right)_{0} Q^{i}=\alpha_{0}+\left(\frac{\partial \alpha}{\partial Q}\right)_{0} Q^{i} .
$$

The terms $\alpha_{0}^{i} \equiv \alpha_{0}$ and $\left(\frac{\partial \alpha^{i}}{\partial Q^{i}}\right)_{0} Q^{i} \equiv\left(\frac{\partial \alpha}{\partial Q}\right)_{0} Q^{i}$ are responsible for the Rayleigh and Raman effects, respectively. Since $Q^{i}=Q_{0}^{i} \cdot \cos \left(\omega^{\prime} t+\psi^{i}\right)$, the effect of the modulating term $\left(\frac{\partial \alpha}{\partial Q}\right)_{0} Q^{i}$ is to change the incident frequency $\omega$ in a scattered Raman frequency $\omega^{\text {Ram }}=\omega \pm \omega^{\prime}$ and to add a completely incoherent Raman phase $\psi^{i}$.

The field $\mathbf{E}_{\mathrm{Vv}}^{i}$ scattered by the molecule $i$ under the action of the local average field $\mathbf{G}^{i}$ is proportional to the induced dipole moment $\boldsymbol{\mu}^{i}=\alpha^{i} \mathbf{G}^{i}$. The vector amplitude $\mathbf{G}_{0}^{i}$ of $\mathbf{G}^{i}$ is proportional to the amplitude $\mathbf{E}_{0}$ of the incident (applied) field $\mathbf{E}$, the proportionality constant being, however, dependent in general on the density $\rho$. The position of $i$ with respect to the source and to the observation point leads to a geometrical modification $\varphi^{i}$ of the phase. All in all one has

$$
\mathbf{E}_{\mathrm{Vv}}^{i}=B(\rho) \alpha^{i} \mathbf{E}_{0} \cos \left(\omega t+\varphi^{i}\right),
$$

where $B(\rho)$ depends on the local average field effect.

The field $\mathbf{E}_{\mathrm{VH}}^{i}$ scattered by the molecule $i$ is due in the first order DID approximation, to the dipole moment modification $\delta \boldsymbol{\mu}^{i}$ that the local fluctuating field $\mathbf{G}_{\mathrm{f} 1}^{i}$ induces in $i$. $\mathbf{G}_{\mathrm{f} 1}^{i}$ is the field generated by the neighbouring molecules $j(\neq i)$ (lying at a distance much smaller than the wave length) polarized by the local average field $\mathbf{G}^{j} \approx \mathbf{G}^{i}$ :

$$
\begin{aligned}
\mathbf{G}_{\mathrm{f} 1}^{i} & =\sum_{j(\neq i)} \hat{S}^{i j} \alpha^{j} \mathbf{G}^{j} \approx\left(\sum_{j(\neq i)} \alpha^{j} \hat{S}^{i j}\right) \mathbf{G}^{i} \\
\delta \boldsymbol{\mu}^{i} & =\alpha^{i} \mathbf{G}_{\mathrm{f} 1}^{i}=\left(\sum_{j(\neq i)} \alpha^{i} \alpha^{j} \hat{S}^{i j}\right) \mathbf{G}^{i}=\delta \hat{\alpha}^{i} \mathbf{G}^{i} \\
\delta \hat{\alpha}^{i} & =\sum_{j(\neq i)} \alpha^{i} \alpha^{j} \hat{S}^{i j} \\
\mathbf{E}_{\mathrm{VH}}^{i} & =B(\rho) \delta \alpha^{i} \mathbf{E}_{0} \cos \left(\omega t+\varphi^{i}\right)=B(\rho) \sum_{j(\neq i)} \alpha^{i} \alpha^{j} S_{13}^{i j} \mathbf{E}_{0} \cos \left(\omega t+\varphi^{i}\right) .
\end{aligned}
$$

In these formulae the tensor $\hat{S}^{i j}$, defined by

$$
S_{\tau \tau^{\prime}}^{i j} \equiv \frac{3\left(x_{\tau}^{i}-x_{\tau}^{j}\right)\left(x_{\tau^{\prime}}^{i}-x_{\tau}^{i}\right)-\left(r^{\prime i}\right)^{2} i_{\tau \tau^{\prime}}}{\left(r^{i j}\right)^{5}}
$$

(where $\tau, \tau^{\prime}=1,2,3, x_{\tau}^{i}$ and $x_{\tau}^{j}$ are the position coordinates of the molecules $i$ and $j$, separated by a distance $r^{i j}$, and $\delta_{\tau \tau^{\prime}}$ is the Kronecker symbol), describes the action of $i$ on $j$ [5]. The cumulated effects of these actions lead to the hyperpolarizability $\delta \hat{\alpha}_{i}$ which modifies the polarizability $\alpha^{i}$ and corresponds to the modification $\delta \mu^{i}$ of $\mu^{i}$. The tensorial character of $\delta \hat{\alpha}_{i}$ accounts for the depolarized scattering.

The scattered intensities $I_{\mathrm{vv}}, I_{\mathrm{vH}}$ are obtained from (2), (6) by summing over $i$, squaring and averaging on the systematic time variation $(\sim \cos \omega t)$ as well as on the stochastic one (related to the position fluctuations by means of the $\hat{S}^{i j}$ and $\varphi^{i}$ ) :

$$
\begin{aligned}
& I_{\mathrm{VV}}=A(\rho) E_{0}^{2} \sum_{i m}\left\langle\alpha^{i} \alpha^{m} \cos \left(\omega t+\varphi^{i}\right)\right.\left.\cos \left(\omega t+\varphi^{m}\right)\right\rangle=\frac{1}{2} A(\rho) E_{0}^{2} \sum_{i m}\left\langle\alpha^{i} \alpha^{m}\right\rangle\left\langle\cos \left(\varphi^{i}-\varphi^{m}\right)\right\rangle \\
& I_{\mathrm{VH}}=A(\rho) E_{0}^{2} \sum_{i j}^{\prime} \sum_{m n}^{\prime}\left\langle\alpha^{i} \alpha^{j} \alpha^{m} \alpha^{n} S_{13}^{i j} S_{13}^{m n} \cos \left(\omega t+\varphi^{i}\right) \cos \left(\omega t+\varphi^{m}\right)\right\rangle= \\
&=\frac{1}{2} A(\rho) E_{0}^{2} \sum_{i j}^{\prime} \sum_{m n}^{\prime}\left\langle\alpha^{i} \alpha^{j} \alpha^{m} \alpha^{n}\right\rangle\left\langle S_{13}^{i j} S_{13}^{m n}\right\rangle\left\langle\cos \left(\varphi^{i}-\varphi^{m}\right)\right\rangle,
\end{aligned}
$$


$A(\rho)$ being a not very sensitive function of $\rho$ and the accent meaning $i \neq j$ and $m \neq n$, respectively. The factorization of the various averages is justified in view of the absence of phase relations between the different types of time variations, systematic and stochastic, contained in the quantities $\alpha^{i}, S_{13}^{i j}, \cos \left(\omega t+\varphi^{i}\right)$, as will be clear from the following discussion. Physically one can say that the elementary process in VH (depolarized) scattering is the double scattering [6] (successive scattering) by a pair of molecules as compared with the simple scattering by a single molecule in VV (polarized) scattering. Correlations between molecules and correlations between pairs of molecules are then the determining statistical factors in VV and VH light scattering respectively.

Now, by substituting the polarizability (1) in (2), (8), one obtains for the Rayleigh and Raman VV scattering :

$$
\begin{aligned}
I_{\mathrm{VV}}^{\mathrm{Ray}} & =\frac{1}{2} A(\rho) E_{0}^{2} \alpha_{0}^{2} \sum_{i m}\left\langle\cos \left(\varphi^{i}-\varphi^{m}\right)\right\rangle \\
I_{\mathrm{VV}}^{\mathrm{Ram}} & =\frac{1}{2} A(\rho) E_{0}^{2}\left(\frac{\partial \alpha}{\partial Q}\right)_{0}^{2} \sum_{i m}\left\langle Q^{i} Q^{m}\right\rangle\left\langle\cos \left(\varphi^{i}-\varphi^{m}\right)\right\rangle .
\end{aligned}
$$

In these formulae the geometrical phases disappear for different reasons. First, the theory of fluctuations gives [7], far enough from the critical point,

$$
\left\langle\sum_{i m} \cos \left(\varphi^{i}-\varphi^{m}\right)\right\rangle=N \frac{R T \chi_{\mathrm{T}}}{V_{\mathrm{M}}}
$$

where $R$ is the ideal gas constant, $T$ the absolute temperature, $\chi_{\mathrm{T}}$ the isothermal compressibility and $V_{\mathrm{M}}$ the molar volume. Second, because of the incoherence of the Raman phases $\psi^{i}$, one has

$$
\left\langle Q^{i} Q^{m}\right\rangle=\left\langle\left(Q^{i}\right)^{2}\right\rangle \delta_{i m}=\left\langle Q^{2}\right\rangle \delta_{i m} .
$$

Therefore :

$$
\begin{aligned}
& I_{\mathrm{VV}}^{\mathrm{Ray}}=\frac{1}{2} A(\rho) E_{0}^{2} \alpha_{0}^{2} \frac{R T \chi_{\mathrm{T}}}{V_{\mathrm{M}}} N \\
& I_{\mathrm{VV}}^{\mathrm{Ram}}=\frac{1}{2} A(\rho) E_{0}^{2}\left(\frac{\partial \alpha}{\partial Q}\right)_{0}^{2}\left\langle Q^{2}\right\rangle N .
\end{aligned}
$$

The same substitution of (1) in (5) yields for the total hyperpolarizability the expression

$$
\delta \hat{\alpha}^{i}=\left(\delta \hat{\alpha}^{i}\right)^{\mathbf{R a y}}+\left(\delta \hat{\alpha}^{i}\right)^{\mathbf{R a m}} .+ \text { negligible terms },
$$

where

$$
\begin{aligned}
\left(\delta \hat{\alpha}^{i}\right)^{\mathrm{Ray}} & =\sum_{j(\neq i)} \alpha_{0}^{i} \alpha_{0}^{j} \hat{S}^{i j}=\alpha_{0}^{2} \sum_{j(\neq i)} \hat{S}^{i j} \\
\left(\delta \hat{\alpha}^{i}\right)^{\mathrm{Ram}} & =\sum_{j(\neq i)}\left[\alpha_{0}^{i}\left(\frac{\partial \alpha^{j}}{\partial Q^{j}}\right)_{0} Q^{j}+\left(\frac{\partial \alpha^{i}}{\partial Q^{i}}\right)_{0} Q^{i} \alpha_{0}^{j}\right] \hat{S}^{i j}=\alpha_{0}\left(\frac{\partial \alpha}{\partial Q}\right)_{0} \sum_{j(\neq i)}\left(Q^{i}+Q^{j}\right) \hat{S}^{i j} .
\end{aligned}
$$

The two terms in (16), $Q^{i} \hat{S}^{i j}$ and $Q^{j} \hat{S}^{i j}$, correspond to the following alternative concerning the mechanism of the double scattering associated with the pair $i j: 1)$ Rayleigh scattering by $j$ followed by Raman scattering by $i$; 2 ) Raman scattering by $j$ followed by Rayleigh scattering by $i$. Then (9) gives

$$
\begin{aligned}
I_{\mathrm{VH}}^{\mathrm{Ray}}=\frac{1}{2} A(\rho) E_{0}^{2} \alpha_{0}^{4} \sum_{i j}^{\prime} \sum_{m n}^{\prime}\left\langle S_{13}^{i j} S_{13}^{m n}\right\rangle\left\langle\cos \left(\varphi^{i}-\varphi^{m}\right)\right\rangle \\
\begin{aligned}
I_{\mathrm{VH}}^{\mathrm{Ram}}=\frac{1}{2} A(\rho) E_{0}^{2} \alpha_{0}^{2}\left(\frac{\partial \alpha}{\partial Q}\right)_{0}^{2} \sum_{i j}^{\prime} \sum_{m n}^{\prime}\left\langle Q^{i} Q^{m}+Q^{i} Q^{n}+Q^{j} Q^{m}+Q^{j} Q^{n}\right\rangle\left\langle S_{13}^{i j} S_{13}^{m n}\right\rangle\left\langle\cos \left(\varphi^{i}-\varphi^{m}\right)\right\rangle= \\
=2 A(\rho) E_{0}^{2} \alpha_{0}^{2}\left(\frac{\partial \alpha}{\partial Q}\right)_{0}^{2}\left\langle Q^{2}\right\rangle \sum_{i j}^{\prime} \sum_{m n}^{\prime} \delta_{i m}\left\langle S_{13}^{i j} S_{13}^{m n}\right\rangle\left\langle\cos \left(\varphi^{i}-\varphi^{m}\right)\right\rangle, \quad\left(17^{\prime}\right)
\end{aligned}
\end{aligned}
$$


since the contributions of the four terms $Q^{i} Q^{m}, Q^{i} Q^{n}, Q^{j} Q^{m}, Q^{j} Q^{n}$ are equal $\left.{ }^{1}\right)$. Now in (17), (17') one may retain only the configurations for which $\varphi^{i} \approx \varphi^{m}$, since for $\varphi^{i} \neq \varphi^{m}$, that is for two relatively distant molecules $i$, $m$, the orientations of the pairs $i j$ and $m n$ are independent, which leads by factorization to

$$
\left\langle S_{13}^{i j} S_{13}^{m n}\right\rangle=\left\langle S_{13}^{i j}\right\rangle\left\langle S_{13}^{m n}\right\rangle=0[2] .
$$

Thus the final general formulae for the depolarized scattering are

$$
\begin{aligned}
I_{\mathrm{VH}}^{\mathrm{Ray}} & =\frac{1}{2} A(\rho) E_{0}^{2} \alpha_{0}^{4} S^{\mathrm{Ray}} \\
I_{\mathrm{VH}}^{\mathrm{Ram}} & =\frac{1}{2} A(\rho) E_{0}^{2} \alpha_{0}^{2}\left(\frac{\partial \alpha}{\partial Q}\right)_{0}^{2}\left\langle Q^{2}\right\rangle S^{\mathrm{Ram}}
\end{aligned}
$$

where

$$
S^{\mathrm{Ray}} \equiv \sum_{i j}^{\prime} \sum_{m n}^{\prime}\left\langle S_{13}^{i j} S_{13}^{m n}\right\rangle, \quad S^{\mathrm{Ram}} \equiv 4 \sum_{\substack{i j n \\(i \neq j, i \neq n)}}\left\langle S_{13}^{i j} S_{13}^{i n}\right\rangle
$$

Following Thibeau [2] and Alder [8], it is convenient to classify the various terms in (20) in doublets (two equivalent possibilities : $S_{13}^{i j} S_{13}^{i j}$ and $\left.S_{13}^{i j} S_{13}^{j i}\right)$, triplets (four equivalent possibilities : $S_{13}^{i j} S_{13}^{i n}(j \neq n)$, $\left.S_{13}^{i j} S_{13}^{m j}(i \neq m), S_{13}^{i j} S_{13}^{m i}(j \neq m), S_{13}^{i j} S_{13}^{j n}(i \neq n)\right)$, and quadruplets $\left(S_{13}^{i j} S_{13}^{m n}\right.$, where all indices are different). Then

$$
S^{\mathrm{Ray}}=2 S^{\mathrm{II}}+4 S^{\mathrm{III}}+S^{\mathrm{IV}}, \quad S^{\mathrm{Ram}}=4 S^{\mathrm{II}}+4 S^{\mathrm{III}},
$$

where

$$
\left.\begin{array}{rl}
S^{\mathrm{II}} & \equiv \sum_{i j}^{\prime}\left\langle\left(S_{13}^{i j}\right)^{2}\right\rangle=N(N-1) s^{\mathrm{II}} \approx N^{2} s^{\mathrm{II}} \\
S^{\mathrm{III}} & \equiv \sum_{i j n}^{\prime}\left\langle S_{13}^{i j} S_{13}^{i n}\right\rangle=N(N-1)(N-2) s^{\mathrm{II}} \approx N^{3} s^{\mathrm{III}} \\
S^{\mathrm{IV}} & \equiv \sum_{i j m n}^{\prime}\left\langle S_{13}^{i j} S_{13}^{m n}\right\rangle=N(N-1)(N-2)(N-3) s^{\mathrm{IV}} \approx N^{4} s^{\mathrm{IV}}
\end{array}\right\}
$$

and

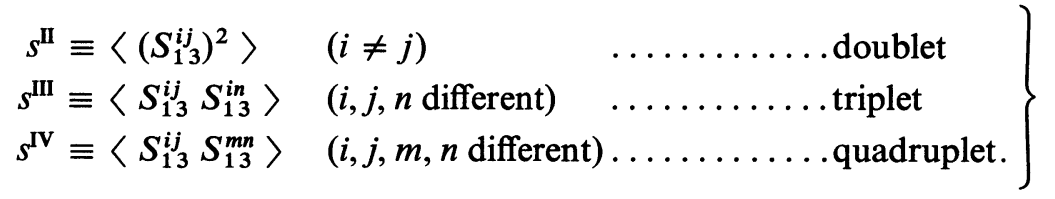

The notations $\sum_{i j}^{\prime}, \sum_{i j m}^{\prime}, \sum_{i j m n}^{\prime}$ are used in the sense of restricting the indices appearing in the sums to values different from one another.

The factor $A(\rho)$ disappears when passing to the depolarizing ratio :

$$
\eta^{\mathrm{Ray}}=\frac{I_{\mathrm{VH}}^{\mathrm{Ray}}}{I_{\mathrm{VV}}^{\mathrm{Ray}}}=\alpha_{0}^{2} \frac{V_{\mathrm{M}}}{R T \chi_{\mathrm{T}}} \frac{1}{N} S^{\mathrm{Ray}}, \quad \eta^{\mathrm{Ram}}=\frac{I_{\mathrm{VH}}^{\mathrm{Ram}}}{I_{\mathrm{VV}}^{\mathrm{Ram}}}=\frac{\alpha_{0}^{2}}{N} S^{\mathrm{Ram}} .
$$

In conclusion, the problem of the depolarized scattering is reduced to the statistical problem of the doublet, triplet, and quadruplet correlations (23), thus proving the fundamental identity of the basic mechanism for this type of scattering in both the Rayleigh and Raman cases. However, to this order of approximation, quadruplet correlations contribute to $I_{\mathrm{VH}}^{\mathrm{Ray}}, \eta^{\mathrm{Ray}}$, but not to $I_{\mathrm{VH}}^{\mathrm{Ram}}, \eta^{\mathrm{Ram}}$.

In contradistinction to the depolarized scattering, the statistical mechanism is not the same for polarized Rayleigh and Raman scattering (see the disappearance of the geometrical phases $\varphi^{i}$ in (10) and (11)).

(') For example, for the terms $Q^{i} Q^{m}$ and $Q^{i} Q^{n}$, one has

since $\hat{S}^{m n}=\hat{S}^{n m}$

$$
\sum_{i j}^{\prime} \sum_{m n}^{\prime} Q^{i} Q^{m} S_{13}^{i j} S_{13}^{m n}=\sum_{i j}^{\prime} \sum_{n m}^{\prime} Q^{i} Q^{n} S_{13}^{i j} S_{13}^{n m}=\sum_{i j}^{\prime} \sum_{m n}^{\prime} Q^{i} Q^{n} S_{13}^{i j} S_{13}^{m n},
$$


The dependence of $\eta^{\mathrm{Ray}}, \eta^{\mathrm{Ram}}$ on the density $\rho$ may be found by direct computation of the sums $S^{\mathrm{II}}, S^{\mathrm{II}}$, $S^{\text {IV }}$ in the low density $(\rho \rightarrow 0)$ and high density $\left(\rho \rightarrow \rho_{\max }\right)$ limits. As will be seen later, the interpolation at intermediate densities seems also possible. One can characterize the maximum density $\rho_{\max }$ in a somewhat vague manner as the density $\rho_{0}$ corresponding to a symmetric and most compact environment, that is to the condensed phase, be it a liquid or a solid phase. Then low density means $\rho / \rho_{0} \ll 1$ and high density means $\rho / \rho_{0} \approx 1$.

Since the calculation of $S^{\mathrm{II}}$, i.e. of $s^{\mathrm{II}}$, reduces to the pair distribution function $g(r)$ problem, it is in principle a soluble problem. Then, by (24), (21), (22), (23), combined Rayleigh and Raman depolarized scattering measures can be used to obtain direct experimental information on $S^{\text {III }}$ and $S^{\text {IV }}$, that is on ternary and quaternary position correlations.

3. The low density limit $\left(\rho / \rho_{0} \ll 1\right)$. - In this limit the terms $s^{\mathrm{II}}, s^{\mathrm{III}}, s^{\mathrm{IV}}$ do not depend on $\rho$, since they are to be computed by means of the $2^{-}, 3^{-}$, and $4^{-}$ particle distribution functions, which at low densities approximately reduce to a density independent pair distribution function $g(r) \approx g_{0}(r)=\mathrm{e}^{-\phi(r) / k T}$, where $\phi(r)$ is the intermolecular potential energy. Then one can neglect $S^{\mathrm{III}}, S^{\mathrm{IV}}$ in comparison with $S^{\mathrm{II}}$ because according to (22) they are proportional to $(N / V)^{2}$, $(N / V)^{3},(N / V)^{4}$, respectively. On the other hand, for an ideal gas,

$$
\chi_{\mathrm{T}} \equiv-\frac{1}{V}\left(\frac{\partial V}{\partial P}\right)_{\mathrm{T}}=\frac{1}{P}=\frac{V_{\mathrm{M}}}{R T}
$$

and thus, from (12), (13), (18), (19), (24) :

$$
\left.\begin{array}{l}
\left.\begin{array}{l}
I_{\mathrm{VV}}^{\mathrm{Ray}}=\frac{1}{2} A(\rho) E_{0}^{2} \alpha_{0}^{2} N, \\
I_{\mathrm{VH}}^{\mathrm{Ray}}=A(\rho) E_{0}^{2} \alpha_{0}^{4} N^{2} s^{\mathrm{II}} \\
\eta^{\mathrm{Ray}}=2 \alpha_{0}^{2} N s^{\mathrm{II}} \sim \rho
\end{array}\right\} \\
I_{\mathrm{VV}}^{\mathrm{Ram}}=\frac{1}{2} A(\rho) E_{0}^{2}\left(\frac{\partial \alpha}{\partial Q}\right)_{0}^{2}\left\langle Q^{2}\right\rangle N, \\
I_{\mathrm{VH}}^{\mathrm{Ram}}=2 A(\rho) E_{0}^{2} \alpha_{0}^{2}\left(\frac{\partial \alpha}{\partial Q}\right)_{0}^{2}\left\langle Q^{2}\right\rangle N^{2} s^{\mathrm{II}} \\
\eta^{\mathrm{Ram}}=4 \alpha_{0}^{2} N s^{\mathrm{II}} \sim \rho .
\end{array}\right\}
$$

The Raman depolarization ratio is twice the Rayleigh one,

$$
\eta^{\mathrm{Ram}} / \eta^{\mathrm{Ray}}=2,
$$

a relation first proposed by Holzer and Le Duff [4]. One has to avoid considering (27) as the consequence of the additivity of the two channels in (16), since the latter concerns the fields only, not the intensities. In fact, (27) loses its validity at higher densities.
4. The high density limit $\left(\rho / \rho_{0} \lesssim 1\right)$. - The only complete treatment of this limit is based on the lattice model (or vacancy model) of Thibeau et al. [1], [3]. (For other model treatments, see [2], [9].) It is supposed that the position correlations can be described by restricting the possible positions of the $N$ molecules to the $N_{0}$ sites of a closed-packed regular lattice that is indeformable and partially occupied, the degree of occupation increasing with the density $\left(N \leqslant N_{0}\right.$; $\left.N / N_{0}=\rho / \rho_{0}\right)$. Alternatively, one can say that the model accounts for the real position fluctuations by means of the occupation fluctuations of a fictitious lattice. While the previous calculations based on the lattice model used the physical, but perhaps somewhat intriguing, concept of the fluctuating polarizability of a lattice site, we give here a formally equivalent but simplified version, which as a matter of fact represents only an approximate computation method of the sums $S^{\mathrm{II}}, S^{\mathrm{III}}, S^{\mathrm{IV}}$.

The method consists of associating with each lattice site $\alpha$ a stochastic (fluctuating) two-valued discret variable $\pi^{\alpha}: \pi^{\alpha}=1$ or $\pi^{\alpha}=0$ according to whether the site is occupied or vacant, respectively. The sum $\sum_{i j}^{\prime}\left(S_{13}^{i j}\right)^{2}$, initially defined on the real instantaneous positions of the molecules, may be rewritten as a sum on the possible (lattice site) $\alpha$ positions, if each term $\left(S_{13}^{i j}\right)^{2}$, written out as $\left(S_{13}^{\alpha \beta}\right)^{2}$, is first multiplied by $\pi^{\alpha} \pi^{\beta}$ :

$$
\left.\sum_{\substack{i j \\ \text { (summed on } \\ \text { the molecules) }}}^{\prime} S_{13}^{i j}\right)^{2}=\sum_{\substack{\alpha \beta \\ \text { (summed on } \\ \text { the lattice sites) }}}^{\prime} \pi^{\alpha} \pi^{\beta}\left(S_{13}^{\alpha \beta}\right)^{2} .
$$

Indeed, the only non-zero terms in (28) are those for which $\pi^{\alpha}=\pi^{\beta}=1$, i.e. those corresponding to occupied site pairs $\alpha \beta$. Therefore

$$
S^{\mathrm{II}}=\sum_{\alpha \beta}^{\prime}\left\langle\pi^{\alpha} \pi^{\beta}\left(S_{13}^{\alpha \beta}\right)^{2}\right\rangle=\sum_{\alpha \beta}^{\prime}\left\langle\pi^{\alpha} \pi^{\beta}\right\rangle\left\langle\left(S_{13}^{\alpha \beta}\right)^{2}\right\rangle_{\sigma}
$$

and, for similar reasons.

$$
\begin{aligned}
& S^{\mathrm{III}}=\sum_{\alpha \beta \delta}^{\prime}\left\langle\pi^{\alpha} \pi^{\beta} \pi^{\delta}\right\rangle\left\langle S_{13}^{\alpha \beta} S_{13}^{\alpha \delta}\right\rangle_{\sigma} \\
& S^{\mathrm{IV}}=\sum_{\alpha \beta \gamma \delta}^{\prime}\left\langle\pi^{\alpha} \pi^{\beta} \pi^{\gamma} \pi^{\delta}\right\rangle\left\langle S_{13}^{\alpha \beta} S_{13}^{\gamma \delta}\right\rangle_{\sigma} .
\end{aligned}
$$

The quantities $\left(S_{13}^{\alpha \beta}\right)^{2}, S_{13}^{\alpha \beta} S_{13}^{\alpha \delta}, S_{13}^{\alpha \beta} S_{13}^{\gamma \delta}$ are defined with respect to fixed points (the lattice points), so that they vary only to the extent that the orientation of the lattice fluctuates (rigid fluctuations), the corresponding averages being denoted by $\langle\cdots\rangle_{\sigma}$. There is no relation between rigid and occupation fluctuations and we therefore factorized the total average. Since rigid fluctuations and their averages do not 
depend on $\rho$, our interest will focus on the occupation Bragg-Williams approximation in order-disorder fluctuations and averages given by $\left\langle\pi^{\alpha} \pi^{\beta}\right\rangle$, $\left\langle\pi^{\alpha} \pi^{\beta} \pi^{\delta}\right\rangle,\left\langle\pi^{\alpha} \pi^{\beta} \pi^{\gamma} \pi^{\delta}\right\rangle$.

theory, the occupation probability $p$ of a site $\alpha$ is

To first order approximation, equivalent to the equal to $N / N_{0}=\rho / \rho_{0}$ independently of the occupation state of the other states. In this approximation

$$
\left.\begin{array}{rl}
\left\langle\pi^{\alpha}\right\rangle & =1 p+0(1-p)=p=\rho / \rho_{0} \\
\left\langle\pi^{\alpha} \pi^{\beta}\right\rangle & =\left\langle\pi^{\alpha}\right\rangle\left\langle\pi^{\beta}\right\rangle=\left(\rho / \rho_{0}\right)^{2} \\
\left\langle\pi^{\alpha} \pi^{\beta} \pi^{\delta}\right\rangle & =\left\langle\pi^{\alpha}\right\rangle\left\langle\pi^{\beta}\right\rangle\left\langle\pi^{\delta}\right\rangle=\left(\rho / \rho_{0}\right)^{3} \\
\left\langle\pi^{\alpha} \pi^{\beta} \pi^{\gamma} \pi^{\delta}\right\rangle & =\left\langle\pi^{\alpha}\right\rangle\left\langle\pi^{\beta}\right\rangle\left\langle\pi^{\gamma}\right\rangle\left\langle\pi^{\delta}\right\rangle=\left(\rho / \rho_{0}\right)^{4}
\end{array}\right\}
$$

the factorization being correct only if the values of the corresponding indices are different.

By (29)-(29") and (30) :

$$
\left.\begin{array}{rl}
S^{\mathrm{II}} & =\left(\frac{\rho}{\rho_{0}}\right)^{2} \sum_{\alpha \beta}^{\prime}\left\langle\left(S_{13}^{\alpha \beta}\right)^{2}\right\rangle_{\sigma}=\left(\frac{\rho}{\rho_{0}}\right)^{2}\left\langle\sum_{\alpha \beta}^{\prime}\left(S_{13}^{\alpha \beta}\right)^{2}\right\rangle_{\sigma} \\
S^{\mathrm{III}} & =\left(\frac{\rho}{\rho_{0}}\right)^{3}\left\langle\sum_{\alpha \beta \delta}^{\prime} S_{13}^{\alpha \beta} S_{13}^{\alpha \delta}\right\rangle_{\sigma} \\
S^{\mathrm{IV}} & =\left(\frac{\rho}{\rho_{0}}\right)^{4}\left\langle\sum_{\alpha \beta \gamma \delta}^{\prime} S_{13}^{\alpha \beta} S_{13}^{\gamma \delta}\right\rangle_{\sigma}
\end{array}\right\}
$$

When finally using the geometrical lattice $\left({ }^{2}\right)$ property [2]

$$
\sum_{\alpha(\neq \beta)} S_{13}^{\alpha \beta}=\sum_{\beta(\neq \alpha)} S_{13}^{\alpha \beta}=0,
$$

the sums $S^{\mathrm{III}}, S^{\mathrm{IV}}$ reduce to $S^{\mathrm{II}}$, since, paying due attention to the meaning of the accents, one has :

$$
\begin{aligned}
& \sum_{\alpha \beta \delta}^{\prime} S_{13}^{\alpha \beta} S_{13}^{\alpha \delta}=\sum_{\substack{\alpha \beta \delta \\
\alpha \neq \beta, \alpha \neq \delta, \beta \neq \delta)}} S_{13}^{\alpha \beta} S_{13}^{\alpha \delta}=\sum_{\substack{\alpha \beta \delta \\
(\alpha \neq \beta, \alpha \neq \delta)}} S_{13}^{\alpha \beta} S_{13}^{\alpha \delta}-\sum_{\alpha \beta}^{\prime} S_{13}^{\alpha \beta} S_{13}^{\alpha \beta}= \\
& =\sum_{\alpha} \sum_{\beta(\neq \alpha)} S_{13}^{\alpha \beta} \sum_{\delta(\neq \alpha)} S_{13}^{\alpha \delta}-\sum_{\alpha \beta}^{\prime}\left(S_{13}^{\alpha \beta}\right)^{2}=-\sum_{\alpha \beta}^{\prime}\left(S_{13}^{\alpha \beta}\right)^{2} \\
& \begin{aligned}
\sum_{\alpha \beta \gamma \delta}^{\prime} S_{13}^{\alpha \beta} S_{13}^{\gamma \delta}=\sum_{\substack{\alpha \beta \gamma \delta \\
\alpha \neq \beta, \gamma, \alpha \neq \delta . \\
\beta \neq \gamma, \beta \neq \delta, \gamma \neq \delta)}} S_{13}^{\alpha \beta} S_{13}^{\gamma \delta}= & \sum_{\substack{\alpha \beta \\
(\beta \neq \gamma, \beta \neq \delta)}}^{\prime} \sum_{\substack{\gamma \delta \\
(\beta \neq \gamma, \beta}} S_{13}^{\alpha \beta} S_{13}^{\gamma \delta}-\sum_{\alpha \beta \delta}^{\prime} S_{13}^{\alpha \beta} S_{13}^{\alpha \delta}-\sum_{\alpha \beta \gamma}^{\prime} S_{13}^{\alpha \beta} S_{13}^{\gamma \alpha}= \\
& =\sum_{\beta \gamma \delta}^{\prime} S_{13}^{\gamma \delta} \sum_{\alpha(\neq \beta)} S_{13}^{\alpha \beta}+\sum_{\alpha \beta}^{\prime}\left(S_{13}^{\alpha \beta}\right)^{2}+\sum_{\alpha \beta}^{\prime}\left(S_{13}^{\alpha \beta}\right)^{2}=2 \sum_{\alpha \beta}^{\prime}\left(S_{13}^{\alpha \beta}\right)^{2} .
\end{aligned}
\end{aligned}
$$

Therefore

$$
\begin{aligned}
S^{\mathrm{II}} / S^{\mathrm{II}} & =-\rho / \rho_{0}, & S^{\mathrm{IV}} / S^{\mathrm{II}} & =2\left(\rho / \rho_{0}\right)^{2} \\
S^{\mathrm{Ray}} & =2\left(1-\frac{\rho}{\rho_{0}}\right)^{2} S^{\mathrm{II}}, & S^{\mathrm{Ram}} & =4\left(1-\frac{\rho}{\rho_{0}}\right) S^{\mathrm{II}}
\end{aligned}
$$

( $\left.{ }^{2}\right)$ By (3), this property is related to the vanishing of the $\mathrm{x}$ component of the local fluctuating field $\mathbf{G}_{\mathrm{fl}}^{\alpha}=\alpha_{0}\left(\sum_{\beta(\neq \alpha)} \hat{S}^{\alpha \beta}\right) \mathbf{G}^{\alpha}$ in Rayleigh scattering. It is, for instance, always obeyed in a cubic lattice : the transformation $x_{1} \rightarrow-x_{1}$ (reflection in the Oyz plane), which should leave the lattice sum

$$
\sum_{\alpha(\neq \beta)} S_{13}^{\alpha \beta}=\sum \frac{3}{\left(\gamma^{\alpha \beta}\right)^{5}}\left(x_{1}^{\alpha}-x_{1}^{\beta}\right)\left(x_{3}^{\alpha}-x_{3}^{\beta}\right)
$$

invariant, obviously changes whereas it sign, so that the sum necessarily vanishes. 
and

$$
\begin{aligned}
& I_{\mathrm{VH}}^{\mathrm{Ray}}=A(\rho) E_{0}^{2} \alpha_{0}^{4}\left(\frac{\rho}{\rho_{0}}\right)^{2}\left(1-\frac{\rho}{\rho_{0}}\right)^{2} \sum_{\alpha \beta}^{\prime}\left\langle\left(S_{13}^{\alpha \beta}\right)^{2}\right\rangle_{\sigma} \\
& I_{\mathrm{VH}}^{\mathrm{Ram}}=2 A(\rho) E_{0}^{2} \alpha_{0}^{2}\left(\frac{\partial \alpha}{\partial Q}\right)_{0}^{2}\left\langle Q^{2}\right\rangle\left(\frac{\rho}{\rho_{0}}\right)^{2}\left(1-\frac{\rho}{\rho_{0}}\right) \sum_{\alpha \beta}^{\prime}\left\langle\left(S_{13}^{\alpha \beta}\right)^{2}\right\rangle_{\sigma} .
\end{aligned}
$$

By (36), (37), the depolarized scattering goes to zero as $\rho \rightarrow \rho_{0}$ (a consequence of the increasing symmetry of the environment of a given molecule) and also as $\rho \rightarrow 0$ (thus joining the low density behaviour (25), (26)). The depolarization ratios are

$$
\begin{aligned}
& \eta^{\mathrm{Ray}}=2 \alpha_{0}^{2} \frac{V_{\mathrm{M}}}{R T \chi_{\mathrm{T}}} \frac{1}{N}\left(\frac{\rho}{\rho_{0}}\right)^{2}\left(1-\frac{\rho}{\rho_{0}}\right)^{2} \sum_{\alpha \beta}^{\prime}\left\langle\left(S_{13}^{\alpha \beta}\right)^{2}\right\rangle_{\sigma} \\
& \eta^{\mathrm{Ram}}=4 \alpha_{0}^{2} \frac{1}{N}\left(\frac{\rho}{\rho_{0}}\right)^{2}\left(1-\frac{\rho}{\rho_{0}}\right) \sum_{\alpha \beta}^{\prime}\left\langle\left(S_{13}^{\alpha \beta}\right)^{2}\right\rangle_{\sigma}=C \frac{\rho}{\rho_{0}}\left(1-\frac{\rho}{\rho_{0}}\right),
\end{aligned}
$$

where $C$ is a constant with respect to $\rho$. Thus, because of the factor $V_{\mathrm{M}} / R T_{\mathrm{T}}$, it is only for $\eta^{\mathrm{Ram}}$ that one can arrive to a definite general conclusion regarding the density dependence. It is worth recalling that the difficulty concerning the density dependence of $\eta^{\text {Ray }}$ can be circumvented [14] by defining a modified Rayleigh depolarization ratio

$$
\left(\eta^{\mathrm{Ray}}\right)_{\text {modified }} \equiv \frac{I_{\mathrm{VH}}^{\mathrm{Ray}}}{I_{\mathrm{VV}}^{\mathrm{Ram}}}=2 \frac{\alpha_{0}^{4}}{(\partial \alpha / \partial Q)_{0}^{2}\left\langle Q^{2}\right\rangle} \frac{1}{N}\left(\frac{\rho}{\rho_{0}}\right)^{2}\left(1-\frac{\rho}{\rho_{0}}\right)^{2} \sum_{\alpha \beta}^{\prime}\left\langle\left(S_{13}^{\alpha \beta}\right)^{2}\right\rangle_{\sigma} \sim \frac{\rho}{\rho_{0}}\left(1-\frac{\rho}{\rho_{0}}\right)^{2} .
$$

An alternative, not equivalent, procedure consists in defining an interference factor $\xi$ which measures the relative contributions of the triplet and quadruplet correlations (with respect to the doublet contribution, prevailing at low densities) :

$$
\begin{aligned}
& \xi^{\mathrm{Ray}} \equiv \frac{2 S^{\mathrm{II}}+4 S^{\mathrm{III}}+S^{\mathrm{IV}}}{2 S^{\mathrm{II}}}=1+2 \frac{S^{\mathrm{II}}}{S^{\mathrm{II}}}+\frac{1}{2} \frac{S^{\mathrm{IV}}}{S^{\mathrm{II}}} \\
& \xi^{\mathrm{Ram}} \equiv \frac{4 S^{\mathrm{II}}+4 S^{\mathrm{III}}}{4 S^{\mathrm{II}}}=1+\frac{S^{\mathrm{III}}}{S^{\mathrm{II}}} .
\end{aligned}
$$

Following (34), one has at high densities

$$
\xi^{\text {Ray }}=\left(1-\frac{\rho}{\rho_{0}}\right)^{2}, \quad \xi^{\text {Ram }}=1-\frac{\rho}{\rho_{0}},
$$

but these formulae do not admit of a direct comparison with the measured quantities, though they can be discussed and tested with reference to the machine calculations in molecular dynamics of Alder et al. [8], see $\$ 6$.

Note also that the simple low density relation (27) is now replaced by

$$
\eta^{\mathrm{Ram}} / \eta^{\mathrm{Ray}}=2 \frac{R T \chi_{\mathrm{T}}}{V_{\mathrm{M}}} \frac{1}{1-\frac{\rho}{\rho_{0}}}
$$

5. The intermediary densities case. - Up to the present the following DID calculations of depolarized (Rayleigh) scattering have been carried out in order to cover the intermediary densities domain :

a) Machine calculations in molecular dynamics [8]. b) Statistical calculations taking into account the triplet term [10]-[12].

The results of $b$ ) are reasonably valid on the low side of the intermediary densities domain. In addition, the problem can be approximately solved by interpolation between the results of $\S 3$ and $\S 4$.

As a matter of fact, the high density formulae (38), (39) join the low density results (25), (26) not only in a qualitative manner $\left(\lim _{\rho \rightarrow 0} \eta^{\mathrm{Ray}}=\lim _{\rho \rightarrow 0} \eta^{\mathrm{Ram}}=0\right)$, but also in a quantitative one

$$
\left(\lim _{\rho \rightarrow 0} \eta^{\mathrm{Ray}}=\frac{1}{2} \lim _{\rho \rightarrow 0} \eta^{\mathrm{Ram}} \sim \rho\right) .
$$

This is a mathematical interpolation argument for extending the formulae (38), (39) to moderate densities. Besides, it seems to us that the following physical reasons exist for this generalization.

The lattice model is justified at high densities in the same sense as the lattice gas model used in Statistical Mechanics since Yang and Lee [13] to study 
phase transitions. However, because of (7), in the optical depolarized scattering problem only the short distance neighbours of a given molecule essentially contribute to the fluctuating local field. Thus, it is the short range order of the possible molecule positions, not the long range one, which gives a qualitatively good description, if any, of the optically important correlations. Moreover, if this is true at high densities, it remains approximately so at any densities, because compact (and hence short range ordered) possible arrangements will probably always prevail for optical reasons even when their statistical weight is low.

Therefore, by (36), (37), (38), (39), (38'), the quantities characterizing the density dependence of the depolarized scattering have a maximum which is to be expected to lie at a higher density in the Raman than in the Rayleigh case.

6. Comparison with experimental results. -6.1 Low DENSITIES. - The low density measurements of $\eta^{\text {Ram }}$ and $\eta^{\text {Ray }}$ [14], [4] show that the ratio $\eta^{\text {Ram }} / \eta^{\text {Ray }}$ is in fact greater than $1\left(\approx 1.3\right.$ for $\mathrm{SF}_{6}, \approx 1.5$ for $\left.\mathrm{CF}_{4}\right)$, but lower than the theoretical value 2 . Since the theoretical value does not depend on the intermolecular potential, this suggests that the discrepancy between theory and experiment reflects the limitations of the DID approximation.

For $\mathrm{SF}_{6}$ and $\mathrm{CH}_{4}$ the measured low density slope of the curve $\eta^{\mathrm{Ram}}$ versus density $\rho$ (in amagat units) at room temperature $(300 \mathrm{~K})$ is $(7 \pm 1) 10^{-5}$ and $(3.5 \pm 0.5) 10^{-5}$, respectively [4]. Using (25) in the form

$$
\left(\eta^{\mathrm{Ram}}\right)_{\mathrm{low}}=\frac{4 \alpha_{0}^{2}}{N} S^{\mathrm{II}},
$$

one obtains, after angular averaging,

$$
\begin{aligned}
\left(\eta^{\mathrm{Ram}}\right)_{\mathrm{low}}=\frac{12}{5} \frac{\alpha_{0}^{2}}{N} \sum_{i j}^{\prime}\left\langle\left(\frac{1}{r^{i j}}\right)^{6}\right\rangle= & \\
& =\frac{12}{.5} \alpha_{0}^{2} \sum_{j(\neq i)}\left\langle\left(\frac{1}{r^{i j}}\right)^{6}\right\rangle
\end{aligned}
$$

and, with the aid of the low density pair distribution function

$$
\begin{gathered}
g_{0}(r)=\exp [-\phi(r) / k T] \\
\left(\eta^{\mathrm{Ram}}\right)_{\text {low }}=\frac{48 \pi}{5} \alpha_{0}^{2} n \int_{0}^{\infty} \frac{1}{r^{4}} \mathrm{e}^{-\phi(r) / k T} \mathrm{~d} r .
\end{gathered}
$$

$n$ is the particle density, the density $\rho$ in amagat units being defined by the ratio $n / n_{0}$, where $n_{0}$ is the particle density under standard conditions. Buckingham and Pople [15] calculated the integrals

$$
\int_{0}^{\infty} r^{2-m} \mathrm{e}^{-\phi(r) / k T} \mathrm{~d} r
$$

for the Lennard-Jones potential and obtained

$$
\left(\eta^{\mathrm{Ram}}\right)_{\text {low }}=\frac{4 \pi}{5} \frac{n_{0} \alpha_{0}^{2}}{\sigma^{3}} \frac{1}{y^{4}} H_{6}(y) \rho
$$

in terms of the tabulated function $H_{m}(y), \sigma$ and $\varepsilon$ being the usual Lennard-Jones parameters and

$$
y \equiv 2(-\varepsilon / T)^{1 / 2} .
$$

From (47) we deduce the low density slope

$$
\left(\frac{\partial \eta^{\mathrm{Ram}}}{\partial \rho}\right)_{\text {low }}=\frac{4 \pi}{5} \frac{n_{0} \alpha_{0}^{2}}{\sigma^{3}} \frac{1}{y^{4}} H_{6}(y) .
$$

The intermolecular potentials for the studied gases are given in table $\mathrm{I}$ and the low density theoretical slopes for $\mathrm{SF}_{6}$ and $\mathrm{CH}_{4}$ at $300 \mathrm{~K}$ are $5.1 \times 10^{-5}$ and $3.1 \times 10^{-5}$, respectively, in reasonably good

\begin{tabular}{|c|c|c|c|c|c|c|c|}
\hline \multirow[b]{3}{*}{ Gas } & \multirow{2}{*}{\multicolumn{2}{|c|}{ Lennard-Jones potential }} & \multirow{3}{*}{$\begin{array}{c}\text { Polarizability } \\
\alpha_{0}\left(\AA^{3}\right)[17]\end{array}$} & \multicolumn{4}{|c|}{$10^{5}(\partial \eta / \partial \rho)_{1 \text { low density }}$} \\
\hline & & & & & Rayleigh & & Raman \\
\hline & $\sigma(\AA)[16]$ & $-\varepsilon(\mathrm{K})[16]$ & & Theory & Experiment & Theory & Experiment \\
\hline $\mathrm{CH}_{4}$ & 3.82 & 148.2 & 2.60 & 2.4 & $\begin{array}{l}1.8 \pm 0.06[21] \\
2.9 \pm 0.4[19] \\
2.6 \pm 0.2[18] \\
2.6 \pm 0.5[23]\end{array}$ & 4.8 & \\
\hline $\mathrm{SF}_{6}$ & 5.51 & 200.5 & 4.47 & 2.6 & $\begin{array}{l}6.1 \pm 0.2[19] \\
9.1 \pm 0.35[20] \\
5.2 \pm 0.07[23]\end{array}$ & 5.1 & $7.1 \pm 1$ \\
\hline $\mathrm{CF}_{4}$ & 4.70 & 152.5 & 2.85 & 1.6 & $2.4 \pm 0.7$ & 3.1 & $3.5 \pm 0.5[4]$ \\
\hline
\end{tabular}
agreement with the experimental values.

6.2 Moderate AND HIGH DENSITIES. - 6.2.1 Comparaison with the numerical results of Alder et al. in molecular dynamics [8]. - Alder et al. calculated the quantities $S^{Z Z}$ and $S_{2}^{Z Z}$ (and, for one particular density, $S_{3}^{Z Z}$ and $S_{4}^{Z Z}$ ), which are connected to the

TABLE I

Low density slopes of $\eta^{\mathrm{Ray}}(\rho)$ and $\eta^{\mathrm{Ram}}(\rho)$ 
previously defined quantities $S^{\mathrm{Ray}}, S^{\mathrm{II}}, S^{\mathrm{III}}, S^{\mathrm{IV}}$ as follows :

$$
\left.\begin{array}{ll}
S^{Z Z}=\frac{4}{3} \frac{\sigma^{6}}{N} S^{\text {Ray }}, & S_{2}^{Z Z}=\frac{4}{3} \frac{\sigma^{6}}{N} S^{\mathrm{II}}, \\
S_{3}^{Z Z}=\frac{4}{3} \frac{\sigma^{6}}{N} S^{\mathrm{III}}, & S_{4}^{Z Z}=\frac{4}{3} \frac{\sigma^{6}}{N} S^{\mathrm{Iv}} .
\end{array}\right\}
$$

The lattice model relates $S^{\text {III }}$ and $S^{\text {IV }}$ to $S^{\text {II }}$ by (34), so that

$$
S_{4}^{Z Z}=2 \frac{\left(S_{3}^{Z Z}\right)^{2}}{S_{2}^{Z Z}} .
$$

The calculations of Alder et al., performed for hard spheres of diameter $\sigma_{0}$ and for a reduced density value of $n^{*}=0,625\left(n^{*} \equiv n \sigma_{0}^{3} / \sqrt{2}\right)$, yielded $2 S_{2}^{Z Z}=10.25$ and $4 S_{3}^{Z Z}=-18.56$. Then, by (50), one has $S_{4}^{Z Z}=8.40$, very close to the molecular dynamics value $S_{4}^{Z Z}=8.52$. Also, by noting that the $2 S_{2}^{Z Z}$ values calculated for 108 and 500 particles are 10.25 and 10.37 , respectively, one can estimate the accuracy of the molecular dynamics result to roughly 0.12 , so that the agreement for $S_{4}^{Z Z}$ turns out to be correct to this order of accuracy. From (50), using (40) and (41), one obtains the following relation between $\xi^{\text {Ram }}$ and $\xi^{\text {Ray }}$,

$$
\xi^{\text {Ram }}=\left(\xi^{\text {Ray }}\right)^{1 / 2}=\left(S^{\text {Ray }} / 2 S^{I I I}\right)^{1 / 2}=\left(S^{Z Z} / 2 S_{2}^{Z Z}\right)^{1 / 2},
$$

which may therefore be considered as approximately verified in molecular dynamics.

6.2.2 Comparison with the experimental results. In order to compare theory with experiment, we have first to estimate $S^{\mathrm{II}}, S^{\mathrm{III}}, S^{\mathrm{IV}}$.

a) If we use the $S^{\mathrm{III}} / S^{\mathrm{II}}$ and $S^{\mathrm{IV}} / S^{\mathrm{II}}$ values predicted by the lattice model, we must first choose a numerical value for $\rho_{0}$. Thibeau [19] found that for gaseous argon and methane at moderate densities an expression like (38) is well verified provided one uses the liquid state density for $\rho_{0}$, while extensive studies of Sung Chung An [22] showed that agreement is obtained for liquefied gases if $\rho_{0}$ equals the solid state density. So there is some doubt about the $\rho_{0}$ value.

b) One could use the Alder's values [8] for $S^{Z Z}$ and $S_{2}^{Z Z}$. Combining the relations (21), (24), and (41), we get

$$
\eta^{\mathrm{Ram}}=\frac{4 \alpha_{0}^{2}}{N} S^{\mathrm{II}} \xi^{\mathrm{Ram}},
$$

which, by using (51), (49), may be written

$$
\eta^{\mathrm{Ram}}=\frac{3 \alpha_{0}^{2}}{2 \sigma^{6}} \sqrt{2 S^{Z Z} S_{2}^{Z Z}} .
$$

For $\eta^{\text {Ray }}$ we obtain in the same way

$$
\eta^{\mathrm{Ray}}=\frac{3 \alpha_{0}^{2}}{4 \sigma^{6}} S^{Z Z} \frac{V_{\mathrm{M}}}{R T \chi_{\mathrm{T}}} .
$$

The values of $S^{Z Z}$ and $S_{2}^{Z Z}$ are tabulated but, unfortunately, most of these calculations use a hard sphere potential of diameter $\sigma_{0}$ which is difficult to relate to the physico-chemical data.

Yet it seems to us that the method $b$ ) is preferable and we propose to choose $\sigma_{0}$ so that the Raman depolarization ratio $\eta^{\mathrm{Ram}}$ have the same value irrespective of the potential used, be it the hard sphere potential $\phi_{0}(r)$ or the Lennard-Jones potential $\phi(r)$. Then, by (46),

$$
\int_{0}^{\infty} \frac{1}{r^{4}} \mathrm{e}^{-\phi(r) / k T} \mathrm{~d} r=\int_{0}^{\infty} \frac{1}{r^{4}} \mathrm{e}^{-\phi o(r) / k T} \mathrm{~d} r,
$$

yielding

$$
\sigma_{0}=\left[\frac{4 y^{4}}{H_{6}(y)}\right]^{1 / 3} \sigma .
$$

Numerical values of $\sigma_{0}$, calculated in this way for $\mathrm{CH}_{4}, \mathrm{CF}_{4}, \mathrm{SF}_{6}$, are given in table II, the reduced density being now defined by $n^{*}=n \sigma_{0}^{3} / \sqrt{2}$.

\begin{tabular}{|c|c|c|c|c|c|c|}
\hline & Gas & $\begin{array}{c}\text { Hard sphere } \\
\text { potential } \\
\sigma_{0}(\AA) \text { at } 300 \mathrm{~K}\end{array}$ & \multicolumn{2}{|c|}{ Rayleigh } & \multicolumn{2}{|r|}{ Raman } \\
\hline & - & - & - & - & - & - \\
\hline Theory & & & 0.22 & 0.58 & 0.50 & 2.66 \\
\hline Experiment & $\mathrm{CH}_{4}$ & $\begin{array}{l}3.36 \\
4.13\end{array}$ & $0.11 \pm 0.02[14]$ & $0.39 \pm 0.06$ & & $188+018[231$ \\
\hline & $\underset{\text { (liquid) }}{\mathrm{SF}_{6}{ }^{4}}$ & 4.72 & & & 0.50 & $1.94 \pm 0.11[4]$ \\
\hline
\end{tabular}

In order to compare the results for different molecules, we introduce a variable $Y$ which is free from equation of state and molecular parameters :

$$
\begin{aligned}
Y^{\mathrm{Ray}} & \equiv \frac{\sigma_{0}^{6}}{\alpha_{0}^{2}} \frac{R T \chi_{\mathrm{T}}}{V_{\mathrm{M}}} \eta^{\mathrm{Ray}} \\
Y^{\mathrm{Ram}} & =\frac{\sigma_{0}^{6}}{\alpha_{0}^{2}} \eta^{\mathrm{Ram}} .
\end{aligned}
$$

TABLE II

Abscissae and ordinates of the maxima of the curves $Y^{\mathrm{Ray}}\left(n^{*}\right)$ and $Y^{\mathrm{Ram}}\left(n^{*}\right)$

Note : the interpretation of the $n^{*}, Y^{\mathrm{Ram}}$ values for liquid $\mathrm{SF}_{6}$ as associated to the maximum is only tentative, see $\S 6.2 .2$. 
By (53) and (54) :

$$
\begin{aligned}
Y^{\mathrm{Ray}} & \equiv \frac{3}{4} S^{Z Z} \\
Y^{\mathrm{Ram}} & =\frac{3}{2} \sqrt{2 S_{2}^{Z Z} S^{Z Z}} .
\end{aligned}
$$

The functions $Y^{\operatorname{Ray}}\left(n^{*}\right), Y^{\mathrm{Ram}}\left(n^{*}\right), \xi^{\mathrm{Ray}}\left(n^{*}\right), \xi^{\text {Ram }}\left(n^{*}\right)$, calculated by (59), (60), (51), (54), with the aid of Alder's values for $S^{Z Z}, S_{2}^{Z Z}$, are represented in figure 2 .

The curves $Y^{\text {Ram }}$ and $Y^{\text {Ray }}$ look very different, in particular the maxima are different. The Raman maximum, much higher than the Rayleigh one (2.66 and 0.58 , respectively), is located at a greater density $\left(n^{*}=0.50\right.$ and $n^{*}=0.22$, respectively). The $Y^{\mathrm{Ram}}$ value is roughly constant for a dense gas ( $Y^{\mathrm{Ram}}$ does not vary more than $10 \%$ between $n^{*}=0.29$ and $n^{*}=0.65 ; n^{*}=0.50$ corresponds to a liquid or a highly compressed gas).

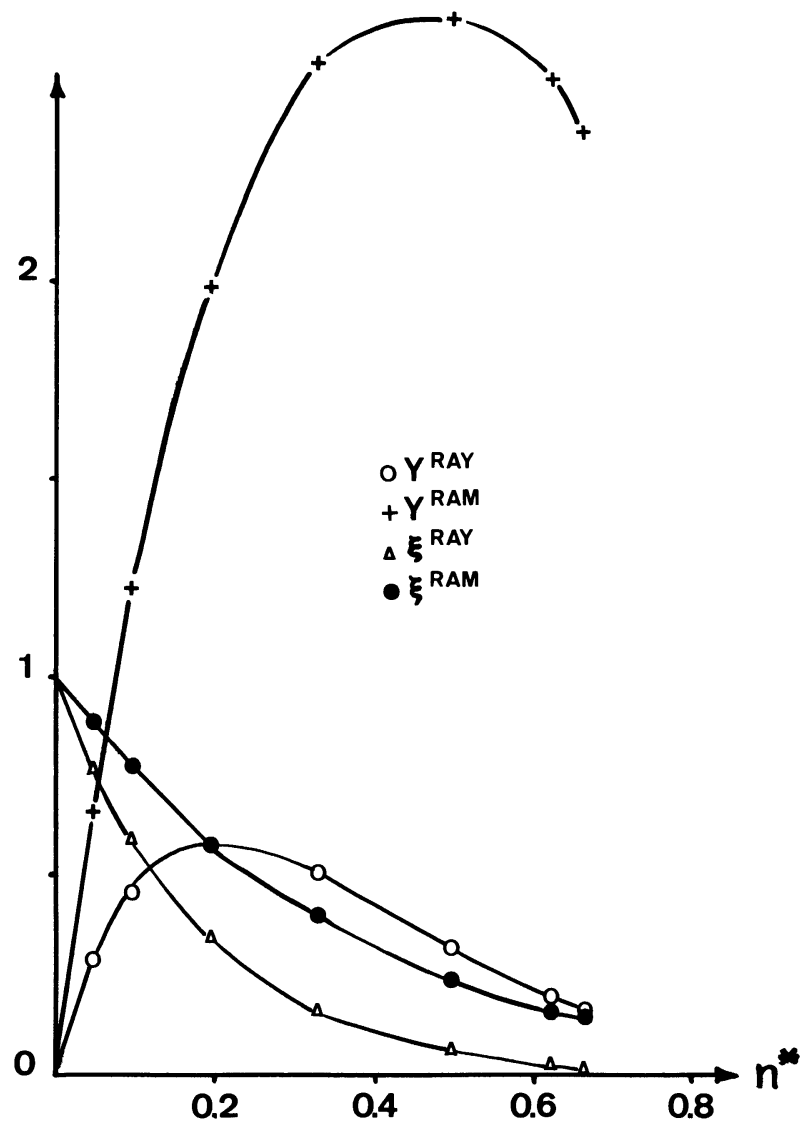

FIG. 2. - The reduced depolarization ratios $Y^{\text {Ray }}, Y^{\text {Ram }}$, see eqs. (57)-(60), and the interference factors $\xi^{\text {Ray }}$, $\xi^{\text {Ram }}$, see eqs. (40), (41), (49), (51), as a function of the reduced density $n^{*}=n \sigma_{0}^{3} / \sqrt{2}$. These quantities have been calculated using the numerical results of Alder et al. [8].
Studying the totally symmetric Raman vibration band of $\mathrm{CF}_{4}$, Gharbi and Le Duff [23] observed for the compressed gas an almost density independent $\eta^{\text {Ram }}$ value, in qualitative agreement with theoretical curve. Their $\eta^{\mathrm{Ram}}=(3.1 \pm 0.3) 10^{-3}$ value corresponds by (58) to $Y^{\mathrm{Ram}}=1.88 \pm 0.18$, which is comparable to the theoretical value. Holzer and Le Duff [4] measured $\eta^{\mathrm{Ram}}$ for liquid $\mathrm{SF}_{6}$, obtaining $\eta^{\mathrm{Ram}}=(3.5 \pm 0.3) 10^{-3}$, which is close to the maximum of the theoretical curve; in this case $\rho \approx 250$, $n^{*} \approx 0.50$. For Rayleigh scattering, Gharbi and Le Duff [14] studied the quantity $\eta^{\mathrm{Ray}} \frac{R T \chi_{\mathrm{T}}}{V_{\mathrm{M}}}$ (apart from a constant factor) versus density and found a maximum about $90 \pm 15$ amagats $\left(n^{*}=0.12 \pm 0.02\right)$ and $150 \pm 30$ amagats $\left(n^{*}=0.11 \pm 0.02\right)$ for $\mathrm{CF}_{4}$ and $\mathrm{CH}_{4}$, respectively, that is at values lower than the theoretical one $\left(n^{*}=0.22\right)$. These values are collected and completed in table II. Thus, the agreement between our theory (supplemented by the molecular dynamics results) and the experiment is more satisfactory for $Y^{\mathrm{Ray}}, Y^{\mathrm{Ram}}$, than for $n^{*}$ corresponding to the maximum of $Y^{\text {Ray }}$. It is difficult to say if this is due to the general limitations of the DID theory or to the much too simplified potential $\phi_{0}(r)$ used in the comparison.

7. Conclusions. - The theory given in this paper connects the collisional DID depolarization effect for Raman and Rayleigh light scattering with the binary, ternary, and quaternary position correlations contained in the doublet, triplet, and quadruplet sums $S^{\mathrm{II}}, S^{\mathrm{III}}, S^{\mathrm{IV}}$. Partial estimation of these sums is possible in the frame of the lattice model, see eq. (34), in terms of the relative density $\rho / \rho_{0}$, where however there remains some uncertainty concerning the precise value of the compact phase density $\rho_{0}$. The relative importance of the high density sums $S^{\mathrm{III}}, S^{\mathrm{IV}}$ with respect to the low density sum $S^{\mathbb{I}}$ is measured by the interference factors $\xi^{\text {Ray }}, \xi^{\text {Ram }}$ defined in (40), (41). By (42), (51), the lattice model unambiguously relates the Raman and Rayleigh scatterings through the relation $\xi^{\text {Ray }}=\left(\xi^{\text {Ram }}\right)^{2}$

Previous theoretical results on Rayleigh scattering are reobtained and the agreement with recent Raman observations is reasonably good. However, in order to improve the comparison with the experimental results, one should : a) include the non-DID depolarizing effects, b) use better intermolecular potentials, and c) calculate $S_{3}^{\mathrm{ZZ}}$ (i.e. $S^{\mathrm{III}}$ ) by molecular dynamics so as to get

$$
\xi^{\mathrm{Ram}}=\frac{S_{2}^{Z Z}+S_{3}^{Z Z}}{S_{2}^{Z Z}}
$$




\section{References}

[1] Thibeau, M., Oksengorn, B., Vodar, B., J. Physique 29 (1968) 287.

[2] Thibeau, M., Oksengorn, B., J. Physique 30 (1969) 47.

[3] Thibeau, M., Tabisz, G. C., OKsengorn, B., Vodar, B., J. Quant. Spectrosc. Radiat. Transfer 10 (1970) 839.

[4] Holzer, W., Le Duff, Y., Phys. Rev. Lett. 32 (1974) 205 ; LE Duff, Y., Thèse Paris (1974).

[5] Landau, L., Lifchitz, E., Théorie des champs (Editions Mir, Moscou) 1970.

[6] Lallemand, P., J. Physique 32 (1971) 119.

[7a] Benoit, H., Stockmayer, W. H., J. Physique 17 (1956) 21.

[7b] Buckingham, A. D., StePhen, M. J., Trans. Faraday Soc. 53 (1957) 884.

[8] Alder, B. J., Weis, J. J., Straus, H. L., Phys. Rev. A 7 (1973) 281.

[9] Dumartin, S., Thibeau, M., Oksengorn, B., C. R. Hebd. Séan. Acad. Sci. B 271 (1970) 884.

[10] Gray, C. G., RalPH, H. I., Phys. Lett. A 33 (1970) 165.

[11] Gelbart, W. M., J. Chem. Phys. 57 (1972) 699.

[12] Mctague, J. P., Ellenson, W. D., Hall, L. H., J. Physique Colloq. 33 (1972) C1-241.
[13] LeE, T. D., Yang, C. N., Phys. Rev. 87 (1952) 410.

[14] Gharbi, A., Le Duff, Y., to be published in Physica.

[15] Buckingham, A. D., Pople, J. A., Trans. Faraday Soc. 51 (1955) 1173.

[16] HiRschfelder, J. O., CURTiss, C. F., BiRD, R. B., Molecular Theory of Gases and Liquids (Wiley, New York) 1954, p. 1110.

[17a] Buckingham, A. D., Dunmur, D. A., Trans. Faraday Soc. 64 (1968) 1776.

[17b] Buckingham, A. D., ORR, B. J., Trans. Faraday Soc. 65 (1969) 673.

[18] Berrue, J., Chave, A., Dumon, B., Thibeau, M., J. Physique 37 (1976) 845.

[19] Watson, R. C., Rowell, R. L., J. Chem. Phys. 61 (1974) 2666.

[20] Triki, A., Oksengorn, B., Vodar, B., C. R. Hebd. Séan. Acad. Sci. 274 (1972) 783.

[21] Thibeau, M., Thèse Paris AO 4302 (1970).

[22] Sung Chung AN, Thesis, Catholic University of Washington (1975).

[23] Gharbi, A., Le Duff, Y., to be published. 